\title{
Pilates e Flexibilidade: Uma Revisão
}

\section{Pilates and Flexibility: A Review}

\author{
JEAN FILIPE ROMÃO PACHECO ${ }^{1}$ \\ ADRIANA COUTINHO DE AZEVEDO GUIMARÃES ${ }^{2}$ \\ MARIA HELENA KRAESKI ${ }^{3}$ \\ ANA CLAUDIA KRAESKI ${ }^{3}$ \\ MELISSA DE CARVALHO SOUZA ${ }^{4}$ \\ CAMILADA CRUZ RAMOS DEARAUJO ${ }^{5}$
}

\section{RESUMO}

Introdução: O método Pilates é considerado uma atividade física eficaz na promoção de saúde, por ser considerada de baixo impacto e de intensidade leve a vigorosa, sendo buscado por diversos grupos com objetivos e necessidades específicas, que comumente envolvem a manutenção da flexibilidade. Objetivo: Esta revisão tem por objetivo identificar e analisar artigos originais que relacionam o método Pilates e a flexibilidade, publicados entre 2002 e 2012 nas línguas portuguesa e inglesa. Material e Métodos: Realizou-se uma busca eletrônica nas bases Google Acadêmico, LILACS, Portal Periódicos da Capes, PubMED e SciELO, até a data limite de 22 de março de 2012. Utilizouse os termos "Pilates" e "flexibilidade" na língua portuguesa e "Pilates" e "flexibility" na língua inglesa. Resultados: Foram identificados 136 estudos com resumo disponível, sendo selecionados 23 artigos que atenderam aos critérios de inclusão, dos quais 12 foram publicados em língua portuguesa e 11 em língua inglesa. Foi verificado que 22 estudos foram realizados com pessoas jovens ou adultas, saudáveis e com patologias músculo-articulares. Conclusão: O método Pilates mostrou-se eficaz não somente na melhora da flexibilidade, como também na resistência muscular, equilíbrio e propriocepção, obtendo resultados relevantes como o aumento da qualidade de vida e a redução do risco de lesões. Todos os estudos relacionados nesta revisão demonstraram que o método Pilates contribui positivamente para a manutenção da flexibilidade dos diferentes grupos investigados, com ganhos significativos em bailarinos.

\section{DESCRITORES}

Técnicas de Exercício e de Movimento. Flexibilidade. Atividade Física.

\begin{abstract}
Introduction: The Pilates method is considered to be an effective physical activity in health promotion, with low impact and mild-tovigorous intensity. Several groups of people have looked for this activity with different purposes and specific needs that usually involve the maintenance of flexibility. Objective: This review aims to identify and analyze studies about increase in flexibility by using the Pilates method of exercise, published between 2002 and 2012, in English and Portuguese . Material and methods: Electronic searches were carried out in the bases Google Scholar, LILACS, Portal Periódicos da Capes, PubMED and SciELO, using the descriptors "Pilates" and "Flexibility" in Portuguese and English. Results: A total of 136 studies with available abstract were retrieved, of which 23 articles met the inclusion criteria and were selected for analysis, being 12 in Portuguese and 11 in English. We found that 22 studies recruited youth or adult participants; eligible subjects were healthy or presented with musculo-articular pathologies. Conclusion: The Pilates method is effective for improving flexibility, as well as muscle resistance, balance and proprioception. It may help obtaining relevant outcomes such as improved quality of life and reduced risk of injury. All of the related studies in this review showed that the Pilates method of exercise contributes positively to the maintenance of flexibility among all groups studied, with significant improvements among ballet dancers.
\end{abstract}

\section{DESCRIPTORS}

Movement and Exercise Techniques. Flexibility. Physical Activity.

1 Educador Físico e Pesquisador do Laboratório de Pesquisa em Lazer e Atividade física do Centro de Ciências da Saúde e do Esporte da Universidade do Estado de Santa Catarina (CEFID/UDESC), Florianópolis, SC, Brasil;

2 Professora Doutora do Departamento de Educação Física e do Programa de Pós-Graduação em Ciências do Movimento Humano do Centro de Ciências da Saúde e do Esporte da Universidade do Estado de Santa Catarina (CEFID/UDESC), Florianópolis, SC, Brasil;

3 Professora Mestre do Departamento de Educação Física do Centro de Ciências da Saúde e do Esporte da Universidade do Estado de Santa Catarina (CEFID/UDESC), Florianópolis, SC, Brasil;

4 Doutoranda do Programa de Pós-Graduação em Ciências do Movimento Humano do Centro de Ciências da Saúde e do Esporte da Universidade do Estado de Santa Catarina (CEFID/UDESC), Florianópolis, SC, Brasil. Bolsista do Programa de Coordenação e Aperfeiçoamento de Pessoal de Nível Superior (CAPES);

5 Pesquisadora do Laboratório de Pesquisa em Lazer e Atividade física do Centro de Ciências da Saúde e do Esporte da Universidade do Estado de Santa Catarina (CEFID/UDESC), Florianópolis, SC, Brasil. 
star fisicamente ativo é um comportamento essencial ao ser humano para a manutenção do seu próprio corpo. Em oposição a esta afirmação, a vida pouco ativa pode ser um risco para a saúde e deve ser evitada. Em meio a estas oposições, que representam uma mesma verdade formal, alguns estudos revelam que é cada vez mais frequente a procura pela atividade física como ferramenta para a promoção de saúde em adultos e idosos ${ }^{1,2}$. Dados da Organização Mundial da Saúde ${ }^{3}$ apontam a atividade física como sendo a melhor ferramenta para a prevenção de doenças crônico-degenerativas e, consequentemente, o envelhecimento. Entretanto, as especificidades do modo de vida atual tornam cada vez mais difíceis manter-se ativo e saudável, visto que as tarefas do dia-a-dia são facilitadas por inovações tecnológicas e pouco tempo é destinado às atividades físicas e de lazer ${ }^{4}$.

A prática regular de atividade física é indicada para a prevenção e tratamento de doenças como osteoporose, diabetes, câncer e cardiopatias, além de proporcionar aumento significativo na qualidade de vida e bem estar psicológico dos praticantes ${ }^{5}$. Observa-se que a atividade física é prevalente em pessoas de 15 a 29 anos de idade e que há uma tendência de redução dos níveis de atividade física com o aumento da idade ${ }^{6,7}$. Os esportes coletivos, caminhada e ginástica são as atividades mais procuradas por pessoas ativas, e os homens são normalmente mais ativos do que as mulheres ${ }^{8,9}$.

Criado pelo alemão Joseph Humbertus Pilates, em 1923, o método Pilates foi introduzido no Brasil na década de $90^{10}$. Trata-se de um método de exercício físico que visa fortalecer e alongar o corpo de maneira global, utilizando movimentos conscientes e baseando-se nos princípios respiração, controle, concentração, organização articular, movimento fluido e precisão ${ }^{11}$. O mesmo autor ressalta que o método foi concebido originalmente para bailarinos e atletas, e vem se tornando bastante popular no Brasil. Pessoas de diferentes grupos e faixas etárias buscam os diversos benefícios deste método, que pode ser aplicado também na reabilitação de pacientes e na manutenção da saúde ${ }^{10}$.

O método Pilates é uma atividade física que pode ser considerada de baixo impacto e de intensidade leve a vigorosa, dependendo do objetivo proposto pelo programa e das individualidades do praticante. Há uma grande quantidade de exercícios e variações que tornam o método Pilates um programa completo de condicionamento físico e mental ideal para diferentes populações ${ }^{10,12,13}$.

De acordo com as novas recomendações do Colégio Americano de Medicina Desportiva, é recomendada a prática regular de exercícios aeróbios e de força, assim como a inclusão de exercícios de flexibilidade na rotina dos praticantes de atividades físicas, considerando a redução expressiva dos riscos e grau de lesão em pessoas que exercitam a flexibilidade de 2 a 3 vezes por semana $^{14}$.

A flexibilidade é uma capacidade motora que relaciona a máxima amplitude de uma articulação com o alongamento máximo dos músculos que cruzam uma determinada articulação, considerando as resistências dos próprios tecidos ${ }^{15}$. Além desta definição, a flexibilidade também é um importante componente na mensuração do nível de aptidão física das pessoas e a sua redução está associada a fatores como idade, sexo e regularidade na prática de atividades físicas ${ }^{16}$, podendo ser evitada por meio da prescrição de exercícios específicos ${ }^{17}$.

Aprimorar a flexibilidade permite que os exercícios sejam executados com maior amplitude de movimento e de maneira mais eficiente. Existem diversas técnicas para desenvolver a flexibilidade, quais sejam: balística, alongamento, estática e facilitação neuromuscular proprioceptiva ${ }^{11}$. O Pilates surge como uma nova técnica para este fim, embora estudos recentes apontem a escassez de evidências científicas que comprovem os benefícios do método, sobretudo em populações sedentárias ${ }^{18-}$ 22 .

Considerando os problemas relacionados à saúde e atividade física, a íntima relação entre o método Pilates e o ganho de flexibilidade de seus adeptos e o crescente número de "estúdios" de Pilates abrigando profissionais da área da Fisioterapia e Educação Física no Brasil, questionase se a produção científica nacional e internacional tem oferecido subsídios que comprovem a eficácia do método Pilates no que se refere ao ganho de flexibilidade. Sendo assim este estudo tem por objetivo identificar e analisar artigos originais que relacionam o método Pilates e a flexibilidade, publicados entre 2002 e 2012 nas línguas portuguesa e inglesa.

\section{MATERIAL E MÉTODOS}

O levantamento bibliográfico que subjaz a 
concepção e redação deste estudo foi baseado em uma revisão das publicações em periódicos nacionais e internacionais até a data limite de 22 de março de 2012. Realizou-se uma busca eletrônica na base/sítio Google Acadêmico e nas bases LILACS (Literatura Latino-Americana e do Caribe em Ciências da Saúde), Portal Periódicos da Capes, PubMED e SciELO (Scientific Electronic Library Online), utilizando os termos "Pilates" e "flexibilidade" na língua portuguesa e "Pilates" e "flexibility" na língua inglesa, presentes no título ou resumo dos artigos, conforme os Descritores em Ciências da Saúde (DeCS).

Os critérios de inclusão foram os artigos originais publicados entre 2002 e 2012, em língua portuguesa e inglesa, em periódicos nacionais e internacionais reconhecidos pela Capes e com Qualis entre "A1" e "B5". Foram excluídos monografias, dissertações, teses, artigos de revisão e estudos que não estavam relacionados diretamente ao tema Pilates e flexibilidade.

Objetivando evitar a perda de quaisquer publicações potencialmente elegíveis, os estudos foram inicialmente analisados por meio do título, resumo e periódico. Os estudos selecionados foram então catalogados de acordo com ano, periódico, Qualis, amostragem, métodos, instrumentos, resultados e conclusão.

\section{RESULTADOS E DISCUSSÕES}

Na Figura 1 é apresentado o fluxograma da estratégia utilizada para a seleção dos estudos.
Foram identificados 136 estudos com resumo disponível, sendo 113 artigos no Google Acadêmico, 7 na base LILACS, 2 no Portal Periódicos da CAPES, 11 na base PubMED e 3 na base SciELO. Analisaram-se título, tema e periódico destes 136 estudos, dos quais 100 foram excluídos por não atenderem aos critérios de inclusão. Os 36 resumos foram então avaliados, sendo que 2 foram excluídos por tratarem-se de revisões sistemáticas e 11 referências foram encontradas em mais de uma base. O número de artigos selecionados para esta revisão foi de 23 , dos quais 17 foram lidos na íntegra e 6 não apresentaram o texto completo disponível online.

Na Tabela 1 são apresentados os detalhes dos estudos selecionados, que incluem os autores, ano de publicação, língua original, periódico, Qualis e base de dados em que foram encontrados.

Dos 23 artigos incluídos nesta pesquisa, $20(87 \%)$ foram publicados a partir de 2006 , além do que não foram encontrados artigos publicados entre 2002 e 2003. Todos os 12 artigos em língua portuguesa foram publicados em periódicos classificados com Qualis "B", e 6 dos 11 artigos em língua inglesa foram publicados em periódicos com Qualis "A".

A análise dos estudos segundo os procedimentos metodológicos previamente estabelecidos está representada na Tabela 2. Todos os estudos analisados envolveram amostras pequenas, variando entre 1 e 60 participantes, perfazendo um total de 572 pessoas estudadas. Houve mais estudos que utilizaram amostras do sexo feminino ou de ambos os sexos

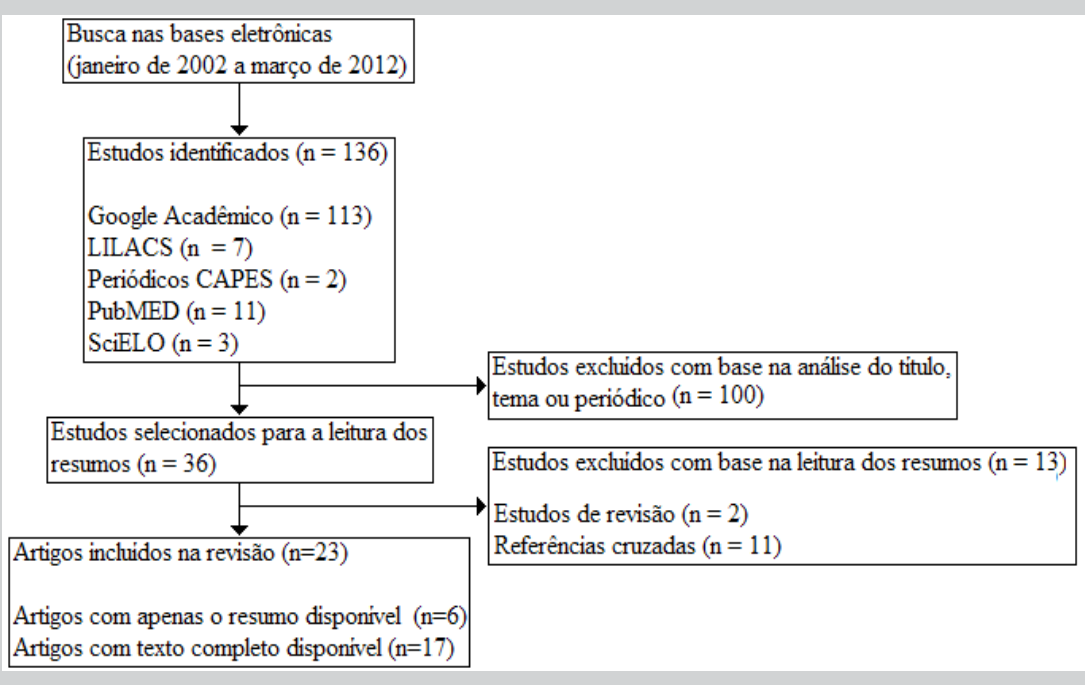

Figura 1 - Fluxograma da seleção dos estudos 
$(=20)^{10,12,13,18,20,21,23,24,27-38}$, enquanto que poucos estudos $(n=2)$ utilizaram amostras do sexo masculino ${ }^{22,25}$.

A maior parte dos estudos foi conduzida com pessoas jovens ou adultas $(n=22)^{10,12,13,18,20-38}$, com um estudo envolvendo idosos ${ }^{21}$. Foram encontrados estudos realizados em pessoas saudáveis $(n=8)^{12,13,22,23,25,31,35,38}$, envolvendo pessoas com patologias músculo-articulares $(n=6)^{18,24,28-30,32}$. O tipo de estudo mais frequente foi o ensaio clínico randomizado $(n=11)^{12,18,21,22,24,25,27,34-37}$, com uma publicação deste tipo em português ${ }^{25}$. Também foram encontrados estudos transversais $(n=7)^{23,26,30-}$ ${ }^{33,38}$ e estudos de caso $(n=5)^{10,13,20,28,29}$, caracterizando a ausência de aleatoriedade na metade das amostras pesquisadas.

Os principais instrumentos utilizados para a avaliação da flexibilidade foram o teste de sentar e alcançar $(n=12)^{20,21,24-27,29,31,32,34,36,38}$, teste de distância do $3^{\circ}$ dedo ao solo $(n=3)^{13,23,28}$, teste de elevação de ombro e punho $(n=2)^{31,34}$, flexímetro $(n=2)^{22,25}$, goniômetro $(n=3)^{13,18,20}$ e avaliações cinemáticas $(n=4)^{10,12,28,35}$. Não foram encontrados estudos que avaliaram a flexibilidade global das amostras por meio de instrumentos como o Flexiteste.

Desconsiderou-se neste estudo o volume, a frequência, os exercícios e os aparelhos utilizados nas intervenções, analisando-se os resultados e conclusões obtidos. Observou-se um aumento da flexibilidade no movimento de flexão de tronco em quase todos os estudos que usaram os testes de sentar e alcançar e de distância do $3^{\circ}$ dedo ao solo 13,20,21,23-29,31,32,34,36,38, um estudo realizado em atletas de futebol não obteve resultados significativos ${ }^{22}$. Todos os resultados obtidos a partir de medidas angulares demonstraram aumento no ângulo de flexão ${ }^{13,18,20,25}$ e extensão ${ }^{20}$ do tronco. Alguns estudos apontam resultados positivos no teste de elevação de ombro e punho ${ }^{31,34}$, entretanto, uma análise cinemática utilizando um sistema de dinamometria comercial (Simulator II) não conseguiu resultados significativos para a articulação do ombro ${ }^{35}$.

Tendo como base os estudos investigados, observou-se que o método vem sendo pesquisado em diferentes grupos como atletas ${ }^{22,25}$, pessoas saudáveis e sedentárias ${ }^{20,21}$ e também em pessoas com problemas músculo-articulares como escoliose $^{18}$, tendinite patelar ${ }^{29}$ e lombalgias ${ }^{24,30,32}$.
Entretanto, os estudos mostram uma tendência em utilizar amostras compostas por mulheres, fato que pode ser explicado pelo maior interesse das mulheres por atividades similares à ginástica, como o Pilates e a loga ${ }^{34}$.

Dos 23 artigos investigados, 17 utilizaram medidas lineares obtidas pelos testes de sentar e alcançar e de distância do $3^{\circ}$ dedo ao solo. A principal vantagem destes testes é o baixo custo e fácil aplicação, porém foram relatados questionamentos e constatações sobre a influência do tronco e do comprimento dos membros nos escores obtidos nestes testes ${ }^{13}$. Foram identificados 11 ensaios clínicos com amostras aleatórias. É importante ressaltar a escassez de pesquisas experimentais na área, sobretudo na língua portuguesa, já que estas pesquisas possibilitam diagnosticar com mais clareza a eficácia do método.

O método Pilates mostrou-se eficaz não somente na melhora da flexibilidade, como também na resistência muscular ${ }^{26}$, equilíbrio ${ }^{21}$ e propriocepção ${ }^{24}$, obtendo resultados relevantes como o aumento da qualidade de vida ${ }^{27,36}$ e a redução do risco de lesões ${ }^{25,35}$. Entretanto, encontrou-se um estudo com atletas de futebol juvenil que concluiu que após um período de treinamento com o método Pilates o aumento da flexibilidade não foi estatisticamente significativo ${ }^{22}$, o que pode talvez sugerir que o método Pilates não seja tão eficaz em determinadas modalidades esportivas ou quando aplicado a adolescentes do sexo masculino.

\section{CONCLUSÃO}

Ao analisar os estudos que compõem esta revisão é possível expor que o método Pilates, na maior parte, contribui positivamente para a manutenção da flexibilidade dos diferentes grupos estudados.

É necessário, no entanto, que novos estudos sejam conduzidos a fim de determinar a influência do método sobre a flexibilidade de amostras mais expressivas e com controle mais rigoroso das variáveis estudadas. Recomenda-se que estudos envolvendo populações jovens e idosas sejam propostos a fim de preencher as lacunas que ainda existem na área, principalmente no Brasil. 


\section{REFERÊNCIAS}

1. Eiras SB, Silva WHA, Souza DL, Vendruscolo R. Fatores de adesão e manutenção da prática de atividade física por parte dos idosos. Rev Bras Ciên Esporte. 2010;31(2):75-89.

2. Santos SC, Knijnik JD. Motivos de adesão à prática de atividade física na vida adulta intermediária. Rev Mackenzie de Educ Fís e Esporte. 2006;5(1):23-34.

3. WHO. Global recommendations on physical activity for health. 2010.

4. Matthews CE, Chen KY, Freedson PS, Buchowski MS, Beech BM, Pate RR et al. Amount of Time Spent in Sedentary Behaviors in the United States, 2003-2004. Am J Epidemiol. 2008;167(7):875-881

5. Warburton DER, Nicol CW, Bredin SSD. Health benefits of physical activity: the evidence. Can Med Assoc J. 2006;174(6):801-809

6. Iser BPM, Claro RM, Moura EC, Malta DC, Morais Neto OL. Fatores de risco e proteção para doenças crônicas não transmissíveis obtidos por inquérito telefônico Vigitel Brasil - 2009. Rev Bras Epidemiol. 2011;14(1Supl):90-102

7. Malta DC, Oliveira MR, Moura EC, Silva SA, Zouain CS, Santos FP et al. Fatores de risco e proteção para doenças crônicas não transmissíveis entre beneficiários da saúde suplementar: resultados do inquérito telefônico Vigitel, Brasil, 2008. Ci \& Saúde Col. 2011;16(3):2011-2022

8. Gonçalves H, Hallal PC, Amorim TC, Araújo CLP, Menezes AMB. Fatores Socioculturais e nível de atividade física no início da adolescência. Rev Panam Salud Publ. 2007;22(4):246-253.

9. Hallal PC, Bertoldi AD, Gonçalves H, Victora CG. Prevalência de sedentarismo e fatores associados em adolescentes de 10-12 anos de idade. Cad Saúde Públ. 2006;22(6):1277-1287.

10. Sacco ICN, Andrade MS, Souza PS, Nisiyama M, Cantuária AL, Maeda FYI, et al. Método pilates em revista: aspectos biomecânicos de movimentos específicos para reestruturação postural - Estudos de caso. Rev Bras Ciên e Mov. 2005;13(4):65-78.

11. Bertolla F, Baroni BM, Leal ECP, Oltramari, JD. Efeito de um programa de treinamento utilizando o método Pilates $®$ na flexibilidade de atletas juvenis de futsal. Rev Bras Med Esporte. 2003;13(4):222-226.

12. Amorim TP, Sousa FM, Santos JAR. Influência de um programa de treino Pilates na força muscular e flexibilidade de bailarinos. Motriz Rev Educ Fís. 2011;17(4):660-666.

13. Miranda LB, Morais PDC. Efeitos do método Pilates sobre a composição corporal e flexibilidade. Rev Bras Prescrição e Fisiologia do Exercício. 2009;3(13):16-21.

14. ACSM. American College of Sports Medicine Position Stand. 2011.

15. Achour Junior A. Alongamento e flexibilidade: definições e contraposições. Rev Bras Atividade Física \& Saúde. 2007;12(1):54-58.

16. Araújo CGS. Avaliação da flexibilidade: valores normativos do Flexiteste dos 5 aos 91 anos de idade. Arq Bras Cardiologia. 2008;90(4):280-287.

17. Dantas EHM, Pereira SAM, Aragão JC, Ota AH. A preponderância da diminuição da mobilidade articular ou da elasticidade muscular na perda da ûexibilidade no envelhecimento. Fitness \& Performance J. 2002;1(3):12-20.
18. Araújo MEA, Silva EB, Mello DB, Cadera SA, Salgado ASI, Dantas EHM. The effectiveness of the Pilates method: Reducing the degree of non-structural scoliosis, and improving flexibility and pain in female college students. J Bodywork \& Mov Ther. 2012;16(2):191-8.

19. Cruz-Ferreira A, Fernandes J, Laranjo L, Bernardo LM, Silva A. A systematic review of the effects of pilates method of exercise in healthy people. Arch Phys Med Rehabilitation. 2011;92(12):2071-2081.

20. Hespanhol Junior LC, Oliveira KTF, Oliveira TGV, Girotto $\mathrm{N}$, Carvalho ACA, Lopes AD. Efeito do método Isostretching na flexibilidade e nível de atividade física em indivíduos sedentários saudáveis. R Bras Ciência e Mov. 2011;19(1):26-31.

21. Irez GB, Ozdemir RA, Evin R, Irez SG, Korkusuz F. Integrating Pilates exercise into an exercise program for $65+$ year-old women to reduce falls. J Sports Scien and Medicine. 2011;10(1):105-111.

22. Pertile L, Vaccaro TC, Marchi T, Rossi RP, Grosseli D, Mancalossi JL. Estudo comparativo entre o método pilates ${ }^{\circledR}$ e exercícios terapêuticos sobre a força muscular e flexibilidade de tronco em atletas de futebol. ConScientiae Saúde. 2011;10(1):102-111.

23. Segal NA, Hein J, Basford JR. The effects of Pilates training on flexibility and body composition: an observational study. Arch of Phys Medicine and Rehabilitation. 2004;85(12):1977-81.

24. Gladwell V, Head S, Haggar M, Beneke R. Does a program of Pilates improve chronic non-speciûc low back pain? Journal of Sports Rehabilitation. 2006;15(4):338-350.

25. Bertolla F, Baroni B, Junior L, Pinto E, Oltramari J. Effects of a training program using the Pilates method in flexibility of sub-20 indoor soccer athletes. Rev Bras Med Esporte. 2007;13(4):222-226

26. Ferreira CB, Aidar FJ, Novaes GS, Vianna JM, Carneiro $A L$, Menezes LS. O método Pilates ${ }^{\circledR}$ sobre a resistência muscular localizada em mulheres adultas. Motricidade. 2007;3(4):76-81.

27. Sekendiz B, Altun Ö, Korkusuz F, Akýn S. Effects of Pilates exercise on trunk strength, endurance and ûexibility in sedentary adult females. J Bodywork \& Mov Ther. 2007;11(4):318-326.

28. Barbosa AC, Maia ESS, Cruz D, Callegary B, Pin AS, Baraúna KMP. Efetividade do método pilates de solo no aumento da flexibilidade. Ter Man. 2009;7(29):21-26.

29. Lara L, Moraes M, Funez EIB. Efeito da prescrição de Pilates na reabilitação da tendinite patelar: estudo de caso. Cinergis. 2009;10(2):28-34.

30. Lima PSQ, Medeiros MSL, Mendes ACG, Laurentino GEC Montenegro EJN. O método Pilates no ganho de flexibilidade dos músculos isquiotibiais em pacientes portadores de hérnia de disco lombar. Fisioter Bras. 2009;10(5):314-317.

31. Rogers K, Gibson AL. Eight-week traditional mat Pilates training-program effects on adult fitness characteristics. Res Quarterly for Exercise and Sport. 2009;80(3):569574. 
32. Rosa HL, Lima JRP. Correlação entre flexibilidade e lombalgia em praticantes de pilates. Rev Mineira de Educ Fís. 2009;17(1):64-73.

33. Silva MCF, Reis FA, Belchior ACG, Pereira DM, Silva BAK, Carvalho PTC. O efeito do treinamento utilizando o método pilates sobre a flexibilidade de musculatura posterior de coxa. Ter Man. 2009;7(31):161-167.

34. Ali AO, Esfarjani F, Bambaeichi E, Marandi M. Poster: The effects of pilates exercise on blood pressure and selective physical fitness components in sedentary overweight females. Brit $\mathrm{J}$ of Sports Medicine. 2010;44(Sup 1):i28.

35. Emery K, De Serres SJ, McMillan A, Côté JN. The effects of a Pilates training program on arm-trunk posture and movement. Clin Biomech. 2010;25(2):124-130.

36. Eyigor S, Karapolat H, Yesil H, Uslu R, Durmaz B. Effects of pilates exercises on functional capacity, flexibility, fatigue, depression and quality of life in female breast cancer patients: a randomized controlled study. European J of Physical and Rehabilitation Medicine. 2010;46(4):481-487.
37. Kloubec J. Pilates for improvement of muscle endurance, flexibility, balance, and posture. J of Strength \& Conditioning Res. 2010;24(3):661-66.

38. Zabott Jr EJ, Ferreira ES. O uso de argolas e argolas associadas a faixas elásticas como forma de atividade dentro das três modalidades do método Pilates. FIEP Bulletin.2010;80.

\section{Correspondência}

Jean Filipe Romão Pacheco

Endereço: Rua Vereador Frederico Veras, 397, Pantanal Florianópolis - Santa Catarina - Brasil

CEP: 88040-200

E-mail: jfrpacheco@yahoo.com.br 